\title{
Relação entre Percepção e Ação durante os Movimentos de Sentar e Levantar em Indivíduos Idosos
}

\author{
Renato de Moraes ${ }^{1}$ \\ Universidade de São Paulo \\ Eliane Mauerberg-DeCastro \\ Universidade Estadual Paulista
}

\begin{abstract}
RESUMO - A proposta deste estudo foi analisar o efeito do envelhecimento nos aspectos perceptivos e motores envolvidos com as ações de sentar e levantar de uma cadeira. Indivíduos jovens e idosos foram filmados enquanto sentavam/levantavam de uma cadeira em sete alturas diferentes do assento. Eles julgaram a dificuldade/facilidade encontrada para sentar e levantar em cada altura do assento. Os idosos exibiram mudanças na estratégia de controle usada para sentar na altura mais baixa do assento e superestimaram o nível de dificuldade/facilidade para realizar as tarefas de sentar e levantar. Em síntese, a percepção de execução fácil da tarefa de sentar pelos idosos não concorda com o grau de dificuldade exibido no desempenho motor na altura mais baixa do assento.
\end{abstract}

Palavras-chave: psicofísica; controle motor; idosos; sentar; levantar.

\section{The Relation between Perception and Action during the Stand-to-Sit and Sit-to-Stand Movements in Elderly Individuals}

\begin{abstract}
The purpose of this study was to analyze aging effects on the perceptual and motor aspects involved in the sit-to-stand and stand-to-sit actions. Young and older individuals were videotaped while performing those actions on a chair adjusted with seven seat heights. Participants estimated, for each seat height, the difficulty/facility perceived while performing the sit-to-stand and stand-to-sit actions. Older individuals exhibited changes in the control strategy used for sitting at the lowest seat height, and overestimated the degree of difficulty/facility involved in performing both actions. In general, the degree of easiness perceived by older individuals at the lowest seat height disagrees with the degree of difficulty exhibited during the motor performance of this task.
\end{abstract}

Keywords: psychophysics; motor control; aged; stand-to-sit; sit-to-stand.

Sentar e levantar de uma cadeira ou de uma cama são apontados como requerimentos básicos para a manutenção de um estilo de vida independente nas pessoas idosas (Ikeda, Schenkman, Riley \& Hodge, 1991; Patla \& Shumway-Cook, 1999; Rodosky, Andriacchi \& Andersson, 1989; Schenkman, Hughes, Samsa \& Studenski, 1996). Essas duas habilidades são classificadas como tarefas de transferência já que envolvem mudanças na posição corporal (Shumway-Cook \& Woollacott, 2007). Na literatura sobre tarefas de transferência existe um grande volume de trabalhos relacionados à ação de levantar (para uma revisão veja Janssen, Bussmann \& Stam, 2002), porém poucos trabalhos interessados com a ação de sentar (Moraes, 1999). A dificuldade na realização dos movimentos de sentar e levantar é um fator de risco que pode predizer a probabilidade de ocorrência de quedas em idosos (Tinetti, Speechley \& Ginter, 1988). O melhor entendimento dos fatores afetando as ações de sentar e levantar em idosos tem implicações clínicas importantes, já que pode ajudar profissionais da saúde a avaliar e organizar programas de prevenção às quedas e de reabilitação.

1 Endereço para correspondência: Escola de Educação Física e Esporte de Ribeirão Preto, Universidade de São Paulo. Av. Bandeirantes, 3900. Ribeirão Preto, SP. CEP 14040-900. Telefone (16) 3602-0522. Fax (16) 3602-0551. E-mail: renatomoraes@usp.br.
As cadeiras disponíveis no mercado possuem assentos com diferentes modelos que incluem variações em altura, tamanho, inclinação, textura da superfície, firmeza, entre outros. A combinação destes atributos físicos é fator crítico no julgamento de quão fácil ou difícil é sentar e levantar da cadeira. De fato, no estudo conduzido por Munton, Ellis, Chamberlain e Wright (1981) sobre adequação de cadeiras, idosos $(n=379)$ consideraram como fatores importantes, em primeiro lugar, a facilidade para levantar, seguido pelo conforto, altura do assento e facilidade para sentar. A facilidade/dificuldade para sentar e levantar de uma cadeira está, provavelmente, muito dependente da variação da altura do assento. Sabe-se que a diminuição da altura do assento aumenta a necessidade de produção de força nos membros inferiores, particularmente na articulação do joelho (Rodosky \& cols., 1989). Esse aumento da demanda de força pode resultar na interpretação subjetiva de que a tarefa é mais difícil de realizar.

O estudo da biomecânica do movimento humano é amplamente usado na área de comportamento motor, especialmente em controle motor. Em geral, estudos sobre as ações de sentar e levantar focam prioritariamente nos aspectos biomecânicos envolvidos com a realização desses movimentos. $\mathrm{O}$ estudo de parâmetros cinemáticos (Mourey, Grishin, d'Athis, Po- 
zzo \& Stapley, 2000; Nuzik, Lamb, VanSant \& Hirt, 1986; Rodosky e cols., 1989; Schenkman, Berger, Riley, Mann \& Hodge, 1990), cinéticos (Hirschfeld, Thorsteinsdottir \& Olsson, 1999; Pai \& Lee, 1994; Pai \& Rogers, 1990, 1991a, 1991b; Rodosky \& cols., 1989) e eletromiográficos (Coghlin \& McFadyen, 1994; Munton, Ellis \& Wright, 1984; Wheeler, Woodward, Ucovich, Perry \& Walker, 1985) contribui para entender os mecanismos de controle usados pelas pessoas jovens e idosas durante as ações de sentar e levantar. Entretanto, tais estudos desvelam uma parte do processo de controle destes movimentos. Por focar em parâmetros biomecânicos, eles ignoram os aspectos perceptivos envolvidos com esses atos motores. Nenhuma ação é puramente motora, mas sim, envolve a combinação dos aspectos perceptivos e motores. Dessa forma, é imperativo entender como se dá a relação entre percepção e ação durante os atos de sentar e levantar. Considerando que a facilidade para sentar e levantar aparece no topo das reivindicações dos idosos em relação ao modelo da cadeira, torna-se necessário desenvolver estudos que avaliem aspectos perceptivos objetivamente.

Em psicofísica, o uso de escalas de categoria é uma ferramenta importante no estudo da percepção em diferentes modalidades sensoriais (Noble \& Robertson, 1996). A combinação da variação na altura do assento com o uso de uma escala de categoria envolvendo os conceitos de dificuldade e facilidade em diferentes níveis permite identificar quais alturas são mais adequadas para indivíduos idosos sentarem/ levantarem.

A percepção de facilidade/dificuldade envolve uma experiência sensorial complexa. Diferentes receptores sensoriais estão envolvidos nessa modalidade sensorial. Os receptores musculares (fusos musculares e órgãos tendinosos de Golgi), articulares e cutâneos fornecem informação sobre a intensidade da atividade muscular e sobre a amplitude de movimento articular durante os movimentos de sentar e levantar. A visão pode contribuir também já que as pessoas olham para a cadeira antes de sentar. Estudos envolvendo a percepção de affordances sugerem que a definição de qual altura do assento permite sentar ou não está diretamente relacionada com a inspeção visual da cadeira e com as dimensões corporais, particularmente com o comprimento dos membros inferiores (Mark, 1987; Mark \& Vogele, 1987). Além disso, durante os movimentos de sentar e levantar a visão contribui para a percepção da posição dos segmentos corporais no espaço e das posições relativas entre eles. Gibson (1979) definiu essa modalidade sensorial como expropriocepção.

A percepção de dificuldade/facilidade para a realização dos movimentos de sentar e levantar pode estar relacionada com alterações nos receptores sensoriais. Se, enquanto levantando, a força é um preditor importante da capacidade de desempenhar o gesto (Schenkman \& cols., 1996), similarmente, a informação somatossensorial dos músculos e articulações pode ser um preditor importante da percepção do grau de dificuldade/facilidade das tarefas de sentar e levantar. Estudos em idosos sobre a percepção da posição articular indicam uma perda na capacidade de identificar o início dos movimentos articulares durante movimento passivo dos membros, bem como uma maior dificuldade em reproduzir o movimento passivo do membro (Kaplan, Nixon, Reitz, Rindfleish \& Tucker, 1985; Skinner, Barrack \& Cook, 1984). As pessoas idosas também exibem dificuldade em detectar a flexão e extensão máxima das articulações metacarpofalangeanas (Kokmen, Bossemeyer \& Williams, 1978).

O objetivo geral do presente estudo foi analisar o efeito do envelhecimento nos aspectos perceptivos e motores envolvidos com as ações de sentar e levantar de uma cadeira. Mais especificamente, o primeiro objetivo deste estudo foi, através de tarefas de julgamento de uma escala de categorias, analisar o impacto do envelhecimento na percepção de dificuldade/facilidade para sentar e levantar de uma cadeira com regulagem do assento para diferentes alturas. $\mathrm{O}$ segundo objetivo foi, durante a posição sentada em uma cadeira com o assento regulado em diferentes alturas, analisar o efeito do envelhecimento na percepção da posição articular dos membros inferiores. O terceiro objetivo foi, através de parâmetros cinemáticos dos movimentos de sentar e levantar de uma cadeira com o assento regulado em diferentes alturas, analisar o efeito do envelhecimento no controle e na coordenação motora destes dois gestos.

\section{Método}

\section{Participantes}

Nove adultos jovens (grupo jovem, GJ) e nove idosos (grupo idoso, GI) foram convidados a participar do presente estudo. Todos os participantes eram fisicamente ativos (i.e., praticavam atividade física regular ao menos três vezes por semana). Os indivíduos do GI participavam do Projeto de Extensão "Atividade Física Para a Terceira Idade," promovido pelo Departamento de Educação Física da Universidade Estadual Paulista (UNESP), campus de Rio Claro. Os participantes do GJ eram alunos de graduação da mesma instituição. Após serem informados sobre os procedimentos envolvidos no experimento, todos eles assinaram um Termo de Consentimento ${ }^{2}$ para participar do estudo. No GJ, os valores médios ( \pm desvio-padrão) para a idade, massa corporal e estatura foram 21,8 anos ( $\pm 1,5), 70,1 \mathrm{~kg}( \pm 11,3)$ e 1,73 m ( \pm 0,13), respectivamente, enquanto que para o GI foram 64,6 anos $( \pm 3,7), 64,3 \mathrm{~kg}( \pm 9,7)$ e $1,60 \mathrm{~m}( \pm 0,07)$, respectivamente.

\section{Procedimento}

Inicialmente foi medida a distância entre a linha média do joelho e o chão para cada participante (i.e., altura padrão). Essa medida foi usada para padronizar as alturas do assento para cada participante. Dessa forma, a variação de estatura entre os participantes não foi uma variável de influência no

2 No período de coleta de dados do presente estudo (i.e., biênio 19961997) não havia um comitê de ética na instituição onde as coletas de dados foram realizadas. De fato, a Resolução $n^{\circ}$. 196, que estabelece as diretrizes e normas regulamentadoras de pesquisas envolvendo seres humanos, somente foi publicada em 10 outubro de 1996. Apesar disso, nós tivemos o cuidado de solicitar aos participantes a assinatura de um termo de consentimento e informamos aos participantes que eles poderiam se retirar do estudo a qualquer momento durante a coleta de dados. Os nomes dos participantes também foram mantidos em sigilo. 
presente estudo. Sete alturas do assento foram testadas para cada participante nas tarefas de sentar e levantar. A escala de estímulos (i.e., alturas do assento), construída seguindo uma progressão geométrica com um quociente igual a 1,26, foi a seguinte: 39,8\% (1), 50,1\% (2), 63,1\% (3), 79,4\% (4), 100\% (5), 125,9\% (6) e 158,5\% (7) da altura padrão. Por exemplo, se a altura padrão fosse $50 \mathrm{~cm}$, as alturas da cadeira seriam 19.9 cm (39,8\%), 25,1 cm (50,1\%), 31,6 cm (63,1\%), 39,7 cm (79,4\%), 50 cm (100\%), 63 cm (125,9\%) e 79,3 cm (158,5\%).

Uma cadeira foi especialmente desenvolvida para a realização do presente estudo. Para tanto, foram removidos os quatro pés de uma cadeira de escritório. Essa cadeira tinha apoio para o tronco, mas não tinha apoio para os braços. Sob o assento foi fundido um macaco hidráulico que permitia facil- mente abaixar ou subir o assento (Figura 1). Esse dispositivo permitiu variar a altura do assento linearmente com o auxílio de uma fita métrica posicionada perpendicularmente ao chão. Entre uma tentativa e outra, a altura do assento foi ajustada sem a presença do participante sobre a cadeira.

Todos os participantes iniciaram a tarefa pelo movimento de sentar. Eles foram solicitados a se posicionar de costas para a cadeira, a uma distância que julgassem suficiente para sentar confortavelmente. A postura inicial envolvia manter o corpo alinhado verticalmente, com os pés paralelos e com os membros superiores repousando ao lado do corpo. Após o comando verbal do experimentador ("pronto"), os participantes podiam iniciar o movimento de sentar a qualquer momento. Os participantes foram informados que deviam manter o con-

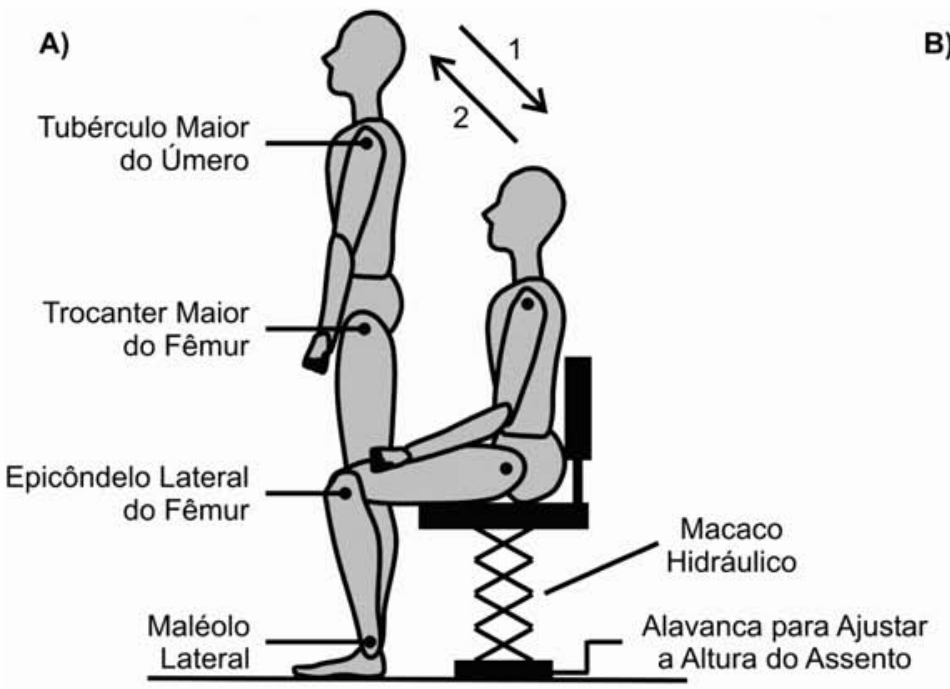

B)

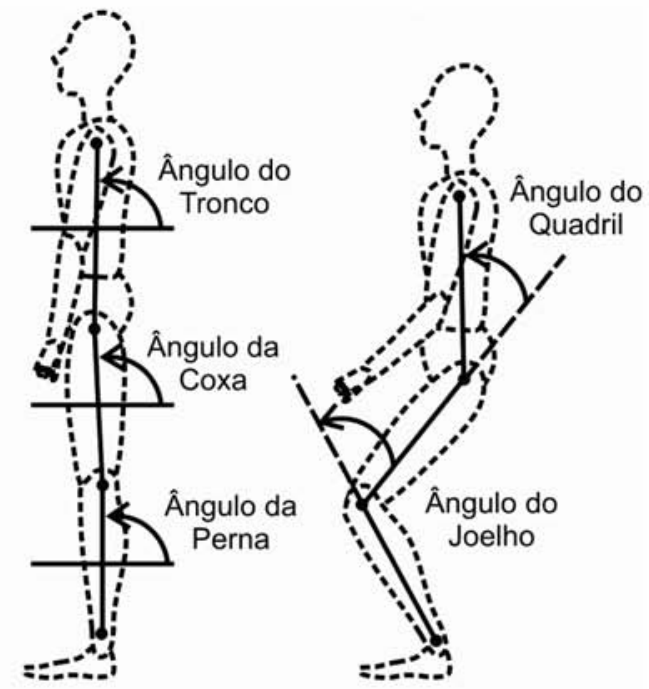

C)
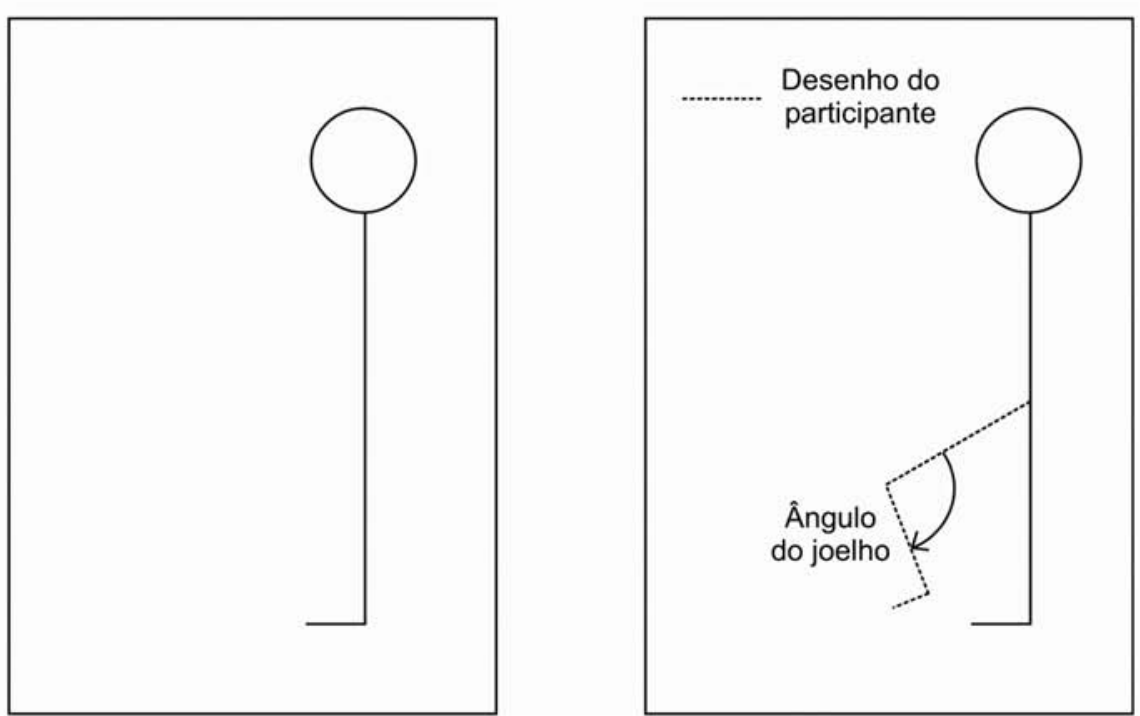

Figura 1. (A) Representação do esquema experimental usado, mostrando a cadeira com o macaco hidráulico adaptado, as posições iniciais e finais dos movimentos analisados, e a posição dos quatro marcadores anatômicos. As setas com os números 1 e 2 representam, respectivamente, os movimentos de sentar e levantar; (B) Representação esquemática dos ângulos calculados para cada um dos segmentos corporais (perna, coxa e tronco) e para as articulações (joelho e quadril). A seta curvada indica a direção em que os ângulos foram calculados; (C) Representação esquemática da folha de papel fornecida ao participante para a tarefa de percepção da posição da articulação do joelho (lado esquerdo). No lado direito, está ilustrado um exemplo hipotético de um desenho feito pelo participante (linha pontilhada) após sentar no assento. 
tato do pé com o chão enquanto sentavam. Após terminarem o movimento de sentar (i.e., tronco verticalmente alinhado), os participantes escolheram em uma escala de categoria numérica, com valores estabelecidos entre 1 e 7 (Tabela 1), o valor que mais se identificava com a dificuldade/facilidade encontrada na realização da tarefa de sentar na altura solicitada (i.e., método psicofísico de estimação de categorias). Para tanto, o experimentador apresentou a escala impressa para os participantes ao final de cada tentativa. Logo após o julgamento perceptivo de dificuldade/facilidade, os participantes foram solicitados a desenhar a posição da sua coxa e perna numa folha de papel que possuía um boneco padrão desenhado para avaliar a percepção da posição articular do joelho (i.e., método psicofísico de produção de magnitude) (Figura 1). Assim que o participante sentava na cadeira, uma prancheta branca $(70$ x $40 \mathrm{~cm})$ era colocada e mantida pelo experimentador acima dos seus segmentos inferiores para evitar que o participante olhasse para baixo antes de desenhar a posição dos seus membros inferiores. Sobre essa prancheta era colocada a folha de papel com o desenho padrão e, então, o participante com um lápis e uma régua (opcional) desenhava a posição em que ele estava percebendo que seus membros inferiores estavam posicionados.

Tabela 1. Escala de percepção do nível de dificuldade/facilidade.

\section{Categorias}

Extremamente Difícil
Muito Difícil
Difícil
Nem Fácil, Nem Difícil
Fácil
Muito Fácil
Extremamente Fácil

Após a realização do desenho, a prancheta era retirada e os participantes eram solicitados a levantar da cadeira. A posição inicial consistia do tronco posicionado verticalmente, com os pés paralelos e com os membros superiores posicionados livremente ao lado do corpo. Após o término do movimento de levantar, os participantes tinham que, da mesma forma, julgar o nível de dificuldade/facilidade dessa tarefa por meio da escala impressa apresentada pelo experimentador. Os participantes foram instruídos a realizar os movimentos de sentar e levantar na sua velocidade preferida. Nas duas tarefas, os participantes podiam usar os membros superiores para auxiliar na realização dos movimentos de sentar e levantar.

Os participantes realizaram dois blocos de tentativas, sendo que cada bloco consistiu de sete estímulos que foram completamente randomizados dentro de cada bloco. Os participantes realizaram um total de 14 tentativas. Entre os blocos de tentativas os participantes tiveram um intervalo de 5 minutos para descanso. Além disso, entre as tentativas também havia um intervalo de descanso de 1 minuto (tempo necessário para ajustar a altura do assento).

Durante a realização dos movimentos de sentar e levantar, os participantes foram filmados (Câmera Panasonic, Modelo AG-2900) no plano sagital esquerdo. Essa filmagem permitiu realizar uma análise cinemática do movimento. A filmadora foi posicionada perpendicularmente ao participante e este foi filmado bem no centro da imagem. Esses procedimentos fo- ram realizados para minimizar os erros de perspectiva. Marcadores feitos de esparadrapo $(9.0$ x $6.0 \mathrm{~cm})$ com um círculo preto pintado no centro foram afixados sobre as articulações monitoradas. Os participantes foram solicitados a comparecer à sessão experimental vestidos com traje de banho (ou uma bermuda de material aderente com uma camiseta de alça), o que permitiu a colocação do centro dos marcadores sobre os seguintes pontos anatômicos: maléolo lateral (tornozelo), epicôndilo lateral do fêmur (joelho), trocanter maior do fêmur (quadril) e tubérculo maior do úmero (ombro) (Figura 1). A frequência de filmagem foi de $30 \mathrm{~Hz}$.

\section{Análise dos dados cinemáticos}

A análise cinemática dos movimentos de sentar e levantar foi conduzida para as alturas de 39,8\% (altura mais baixa), $100 \%$ (altura padrão) e 158,5\% (altura mais alta). Somente as tentativas de um dos blocos foram analisadas. O bloco foi definido por meio de sorteio. Somente essas três alturas foram analisadas, já que o estudo piloto indicou que nessas três alturas as diferenças eram mais evidentes e as alturas intermediárias não destacaram informações claras quanto a possíveis diferenças no controle motor.

Um vídeo cassete de quatro cabeças com reprodução de imagem quadro a quadro (Panasonic, Modelo AG-7300) foi conectado a uma placa de captura de imagens em um microcomputador (486DX-33, 128K 212MEG). Com o auxílio do programa 2-D Biomechanical Analysis, as imagens foram reproduzidas na tela do computador. Esse programa permitiu também a obtenção quadro a quadro das coordenadas $\mathrm{x}$ (antero-posterior) e y (vertical) de cada um dos quatro pontos anatômicos (tornozelo, joelho, quadril e ombro). As coordenadas dos marcadores articulares foram filtradas usando um filtro digital de Butterworth passa baixa de $4^{\text {a }}$. ordem com uma frequência de corte de $5 \mathrm{~Hz}$ (Winter, 2005).

A partir da suavização das coordenadas x e y, foram calculados os ângulos ao longo do tempo dos segmentos corporais perna, coxa e tronco em relação à linha horizontal (Figura 1). O cálculo do ângulo dos segmentos foi feito utilizando a Equação 1 abaixo (Winter, 2005):

$$
\theta_{i j}(\text { segmento })=\arctan \frac{y_{j}-y_{i}}{x_{j}-x_{i}} \quad \text { Equação } 1
$$

onde $\theta_{\mathrm{ij}}$ é a posição angular do segmento, $y_{\mathrm{j}}$ e $x_{\mathrm{j}}$ são as coordenadas da articulação superior do segmento, e $y_{\mathrm{i}}$ e $x_{\mathrm{i}}$ são as coordenadas da articulação inferior do segmento. A diminuição do ângulo representa uma aproximação do segmento em relação à linha horizontal e vice-versa para um aumento do ângulo do segmento. Com base nos ângulos dos segmentos, foi possível calcular o ângulo das articulações do joelho e quadril conforme a Equação 2 abaixo:

$$
\theta_{\text {articulação }}=\theta_{1}-\theta_{2} \quad \text { Equação } 2
$$


onde $\theta_{\text {articulacão }}$ é o ângulo da articulação, $\theta_{1}$ é o ângulo do segmento proximal e $\theta_{2}$ é o ângulo do segmento distal (no caso da articulação do joelho, $\theta_{1}$ é o ângulo da coxa e $\theta_{2}$ o ângulo da perna). $\mathrm{O}$ aumento no valor do ângulo indica a existência de movimento de flexão e vice-versa para o movimento de extensão. A velocidade angular e a aceleração angular dos três segmentos e das duas articulações foram calculadas como a primeira e segunda derivadas da posição angular em função do tempo, por meio do método da diferença central (Winter, 2005).

Os movimentos de sentar e levantar foram divididos em duas fases. Para o movimento de sentar, a fase 1 compreendeu o período entre o início do movimento e o contato com o assento da cadeira (fase de abaixamento). A fase 2 do movimento de sentar compreendeu o intervalo de tempo entre o contato com o assento e o término do movimento (fase de estabilização). Para o movimento de levantar, a fase 1 compreendeu o período entre o início do movimento e a perda de contato com o assento (fase de geração do momento linear). A fase 2 do movimento de levantar compreendeu o intervalo entre a perda de contato com o assento e o término do movimento (fase de estabilização). Todos estes eventos foram definidos a partir da inspeção visual quadro a quadro das imagens. A duração total e de cada uma das fases dos movimentos de sentar e levantar foram calculadas contandose o número de quadros e multiplicando esse número pela duração de cada quadro (1/30 ou 0,033 s).

A obtenção das curvas de aceleração permitiu o cálculo da variável unidade de movimento (Clearfield, Diedrich, Smith \& Thelen, 2006). Cada ciclo de aceleração e desaceleração constitui uma unidade de movimento. Assim, para cada um dos segmentos e articulações monitorados foi feita a análise do número de vezes que a curva da aceleração angular cruzou o eixo zero (i.e., $0^{\circ} / \mathrm{s}^{2}$ ) durante a realização dos movimentos de sentar e levantar. $\mathrm{O}$ aumento do número de cruzamentos da curva da aceleração sobre o eixo zero é um indicativo de menor fluidez na realização do movimento e, consequentemente, uma maior dificuldade para controlar apropriadamente a ação.

\section{Análise dos dados psicofísicos}

Para a análise da percepção da posição articular do joelho foi utilizada a medida do ângulo do joelho desenhada pelo sujeito (Figura 1) e a medida capturada pela filmagem. A medida do desenho do ângulo do joelho foi feita utilizando um transferidor. Para coincidir com o mesmo ângulo obtido a partir do desenho, o cálculo do ângulo do joelho, obtido por meio da filmagem no instante em que o participante realizava o desenho, foi feito conforme a Equação 3 abaixo:

$$
\theta_{\text {joelho }}=180-\left(\theta_{\text {coxa }}-\theta_{\text {perna }}\right) \quad \text { Equação } 3
$$

onde $\theta_{\text {coxa }}$ e $\theta_{\text {perna }}$ são os valores da posição angular da coxa e da perna e $\theta_{\text {joelho }}$ é o ângulo da articulação do joelho.

Os valores do ângulo do joelho medidos por meio da filmagem e do desenho foram usados para o cálculo do expoente (n) e da constante escalar (k) da função de potência
(Stevens, 1975). Além dessas variáveis, foi calculado o coeficiente de determinação $\left(\mathrm{r}^{2}\right)$, que corresponde ao valor da correlação de Pearson (r) elevado ao quadrado. Segundo DaSilva e Macedo (1983), o expoente é um índice de sensibilidade perceptiva. Essa sensibilidade está diretamente relacionada com o tamanho do expoente. Um expoente igual a 1,0 significa que a função potência se reduz a uma relação linear entre estímulo e resposta (i.e., constância perceptiva). De outro lado, expoentes menores que 1,0 indicam uma subconstância perceptiva, a qual se revela numa magnitude de resposta desacelerando ao longo de uma escala linear de estímulos. Em algumas modalidades perceptivas, a subconstância perceptual associa-se a uma menor sensibilidade por parte do observador. Quando o expoente é maior do que 1,0 ocorre, ao contrário, uma superconstância perceptiva que é marcada pela aceleração na magnitude da resposta ao longo da escala dos estímulos.

\section{Análise estatística}

Análises de variância (ANOVAs) para dois fatores (2 grupos $\mathrm{x} 7$ alturas do assento) com medidas repetidas para o segundo fator foram realizadas para a variável dependente duração do sentar e do levantar (fase 1, fase 2 e total). Para as variáveis psicofísicas (expoente e constante escalar) e para o coeficiente de determinação, ANOVAs para dois fatores (2 grupos $\mathrm{x} 2$ tentativas) com medidas repetidas no segundo fator foram realizadas. Testes a posteriori de Bonferroni foram realizados para identificar onde residiram as diferenças quando efeitos principais foram observados. Para as interações, testes $t$-student foram conduzidos utilizando o ajuste de Bonferroni (0,05/número de comparações pareadas).

Para o número de cruzamentos da curva de aceleração sobre o eixo zero, o teste não-paramétrico de Mann-Whitney foi realizado para cada segmento/articulação analisado nos movimentos de sentar e levantar nas alturas 1, 5 e 7 . Para os valores de percepção de dificuldade/facilidade o mesmo teste estatístico não-paramétrico foi realizado. Para essas duas variáveis foi computada a mediana e gerados boxplots. Para todas as análises estatísticas realizadas o valor do $\alpha$ foi de 0,05 .

\section{Resultados}

\section{Duração do movimento}

A ANOVA para dois fatores (grupo x altura do assento) com medidas repetidas no segundo fator identificou efeito principal de grupo $\left[F_{(1,16)}=6,149, p=0,025\right]$ e altura do assento $\left[F_{(6,96)}=32,060, p<0,001\right]$, para a duração da fase 1 do movimento de sentar. Para a duração fase 2, a ANOVA apontou efeito principal de grupo $\left[F_{(1,16)}=5,866, p=0,028\right]$ e de altura do assento $\left[F_{(6,96)}=10,204, p<0,001\right]$. A ANOVA para a duração total exibiu também efeito principal de grupo $\left[F_{(1,16)}=7,508, p=0,015\right]$ e de altura do assento $\left[F_{(6,96)}=\right.$ $5,835, p<0,001]$. A duração do sentar foi maior para o GI em comparação ao GJ nas duas fases e no movimento todo 
(Figura 2). Os testes a posteriori de Bonferroni identificaram diferenças significativas entre os pares de altura do assento para a fase 1 do sentar $(1 \times 3,1 \times 4,1 \times 5,1 \times 6,1 \times 7,2 \times 3,2 \times 5$, 2x6, 2x7, 3x5, 3x6, 3x7, 4x5, 4x6, 4x7, 5x6) e fase 2 do sentar (1x5, 1x6, 2x5, 2x6, 3x5, 3x6, 4x5, 4x6). Apesar do efeito principal de altura para a duração total, o teste a posteriori de Bonferroni não identificou nenhuma diferença significativa entre os pares de altura do assento. O aumento da altura do assento resultou, em geral, em uma diminuição da duração da fase 1 e um aumento da duração da fase 2. Para a duração do sentar nas fases 1 e 2, a interação entre grupo e altura do assento foi significativa [Fase 1: $F_{(6,96)}=2,533, p=0,026$; Fase 2: $F(6,96)=2,564, p=0,024]$. Testes t-student [com ajuste de Bonferroni $(0,05 / 7=0.007)]$ identificaram, na fase 1 , um aumento na duração do sentar para o GI na altura 3, $\left[t_{(16)}=3,278, p=0,005\right]$ em comparação ao GJ. Na fase 2, por sua vez, o GI exibiu maior duração para o movimento de sentar na altura $4\left[t_{(16)}=3,277, p=0,005\right]$ e altura $5\left[t_{(16)}\right.$ $=3,476, p=0,003$ ] do que o GJ.
Para o movimento de levantar, a ANOVA para dois fatores (grupo $\mathrm{x}$ altura do assento) com medidas repetidas no segundo fator identificou efeito principal de grupo somente para a duração da fase 1 [ $\left.F_{(1,16)}=12,776, p=0,003\right]$, enquanto que houve efeito principal de altura do assento para a duração da fase $1\left[\left(F_{(6,96)}=7,099, p<0,001\right]\right.$, duração da fase $2\left[F_{(6,96)}\right.$ $=48,923, p<0,001]$ e para a duração total $\left[\left(F_{(6,96)}=18,808\right.\right.$, $p<0,001]$. O GI exibiu uma duração maior da fase 1 em comparação ao GJ (Figura 2). Os testes a posteriori de Bonferroni identificaram diferenças significativas entre os pares de altura para a fase 1 do levantar $(1 \times 6,2 \times 6,3 \times 6,4 \times 6)$, fase 2 do levantar $(1 \times 3,1 \times 4,1 \times 5,1 \times 6,1 \times 7,2 \times 5,2 x 6,2 \times 7,3 \times 5$, $3 \times 6,3 \times 7,4 \times 5,4 \times 6,4 \times 7,5 \times 6)$ e total do levantar $(1 \times 3,1 \times 4$, $1 \times 5,1 \times 6,1 \times 7,2 \times 3,2 \times 5,2 \times 6,2 \times 7,3 \times 7,4 \times 7)$. O aumento da altura do assento gerou, em geral, um aumento da duração da fase 1 e uma diminuição da duração da fase 2 e da duração total. Para a duração da fase 2 houve interação significativa para grupo e altura do assento $\left[F_{(6,96)}=5,409, p<0,001\right]$. Para a duração total também houve interação significativa

\section{Duração do Sentar (s)}

Fase 1

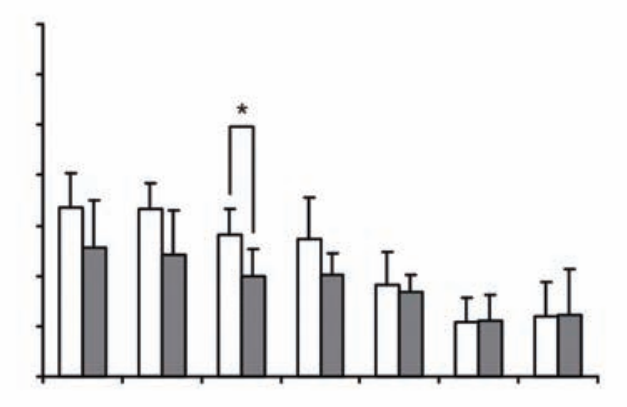

Fase 2
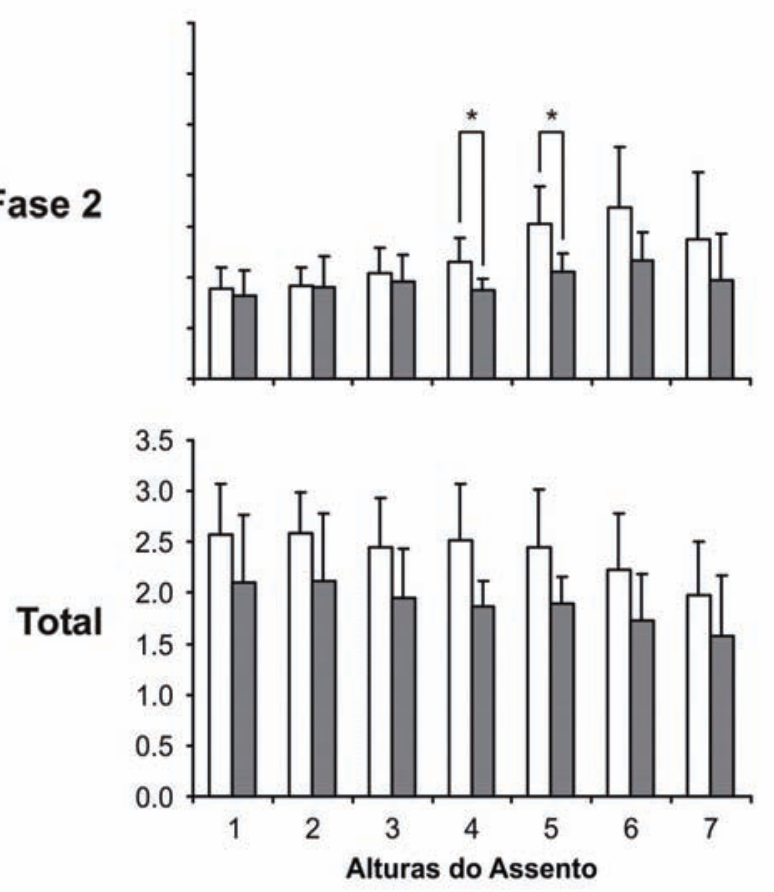

\section{Duração do Levantar (s)}
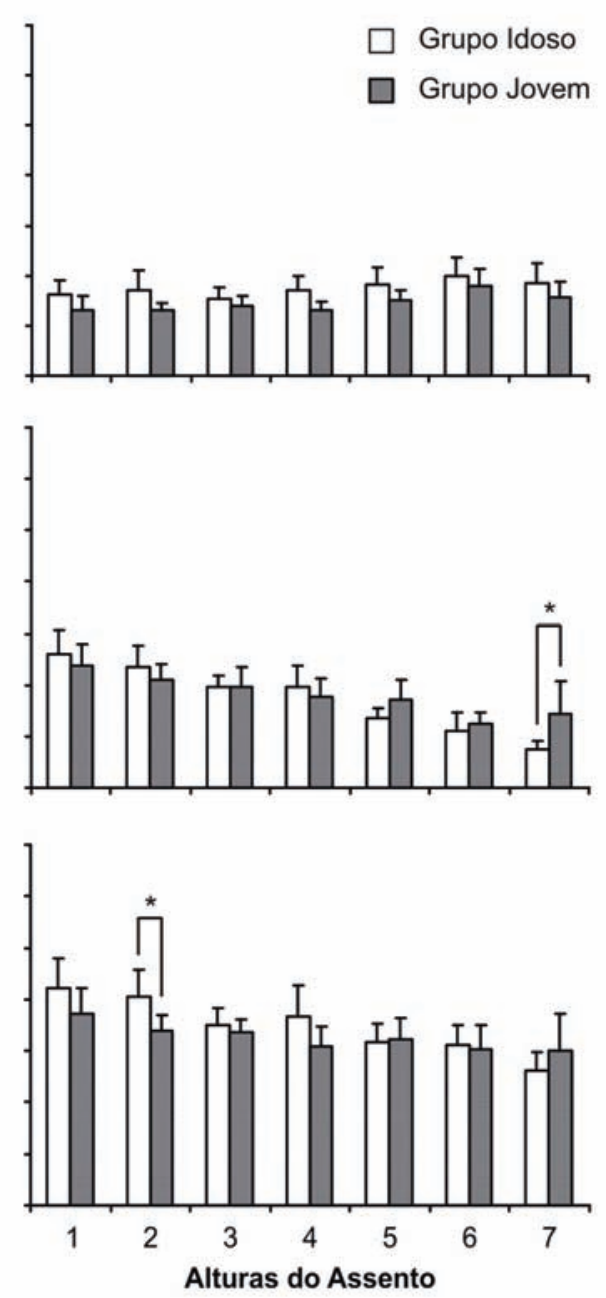

Figura 2. Média e desvio-padrão da duração do sentar (coluna da esquerda) e do levantar (coluna da direita) em segundos (s) para as fases 1 (topo), 2 (meio) e total (em baixo). $(* \mathrm{p} \leq 0.007)$ 
para grupo e altura do assento $\left[F_{(6,96)}=4,417, p=0,001\right]$. O GI exibiu uma menor duração da fase 2 na altura $7\left[t_{(16)}=\right.$ 3,135, $p=0,006$ ] do que o GJ. Na duração total, a diferença entre os grupos residiu na altura $2\left[t_{(16)}=3,186, p=0,006\right]$, sendo que o GI exibiu uma maior duração do que o GJ.

\section{Unidades de movimento}

Para o movimento de sentar, o teste de Mann-Whitney identificou um aumento significativo no número de unidades de movimento para o GI em comparação ao GJ (Figura 3). Na altura 1, as diferenças foram significativas para os segmentos perna $(p=0,008)$ e coxa $(p=0,017)$ e para as articulações do joelho $(p=0,002)$ e quadril $(p=0,021)$. Na altura 5 , as diferenças foram significativas para os segmentos perna $(p=0,015)$, coxa $(p=0,033)$ e tronco $(p=0,018)$ e para a articulação do joelho ( $p=0,037)$. Na altura 7 , as diferenças foram significativas para os segmentos perna $(p=0,021) \mathrm{e}$ tronco $(p=0,008)$ e para as articulações do joelho $(p=0,016)$ e quadril $(p=0,005)$.

Por outro lado, as diferenças foram praticamente inexistentes entre o GI e o GJ para o movimento de levantar. Na altura 1, o teste de Mann-Whitney identificou diferenças no segmento coxa ( $p=0,021)$ e na articulação do joelho ( $p$
$=0,005)$, sendo que nos dois casos houve um aumento no número de unidades de movimento para o GI (Figura 3).

\section{Percepção de dificuldade/facilidade}

Para a percepção de dificuldade/facilidade para o movimento de sentar nas 7 alturas avaliadas, nenhuma diferença entre os grupos foi observada (Figura 4). Para o movimento de levantar, diferenças foram observadas somente para a primeira tentativa nas alturas $2(p=0,026)$ e $3(p=0,003)$. Nos dois casos, o GJ julgou as tarefas como sendo mais fáceis em comparação ao GI.

\section{Percepção da posição articular do joelho}

AANOVA para dois fatores (grupo x tentativa) com medidas repetidas para o segundo fator identificou efeito principal de grupo para as variáveis constante escalar $\left[F_{(1,16)}=8,001\right.$, $p=0,012]$ e expoente da função de potência $\left[F_{(1,16)}^{(1,16)}=7,417\right.$, $p=0,015]$. A constante escalar foi maior para os indivíduos do grupo idoso $(M=0,62, S D=0,19)$ em comparação aos adultos jovens $(M=-0,07, S D=0,76)$. O expoente foi maior para os adultos jovens $(M=1,02, S D=0,38)$ em comparação
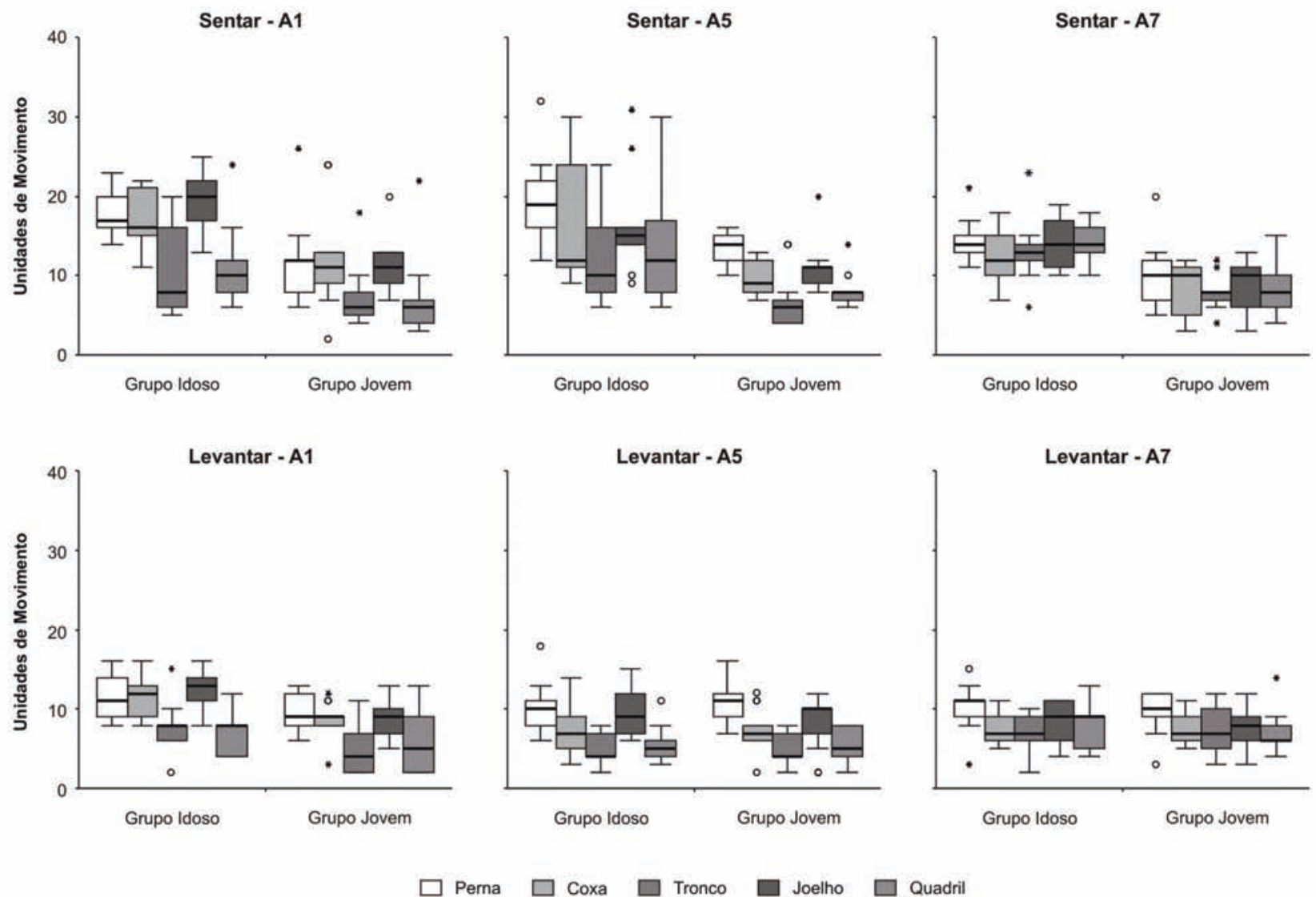

Figura 3. Boxplots das unidades de movimento dos segmentos e articulações analisados. O símbolo $\circ$ representa os casos com valores entre 1,5 e 3,0 vezes o comprimento do retângulo do boxplot a partir da margem inferior ou superior do retângulo. O símbolo * representa os casos com valores acima de 3,0 vezes o comprimento do retângulo do boxplot a partir da margem inferior ou superior do retângulo (GI: grupo idoso | GJ: grupo jovem). 

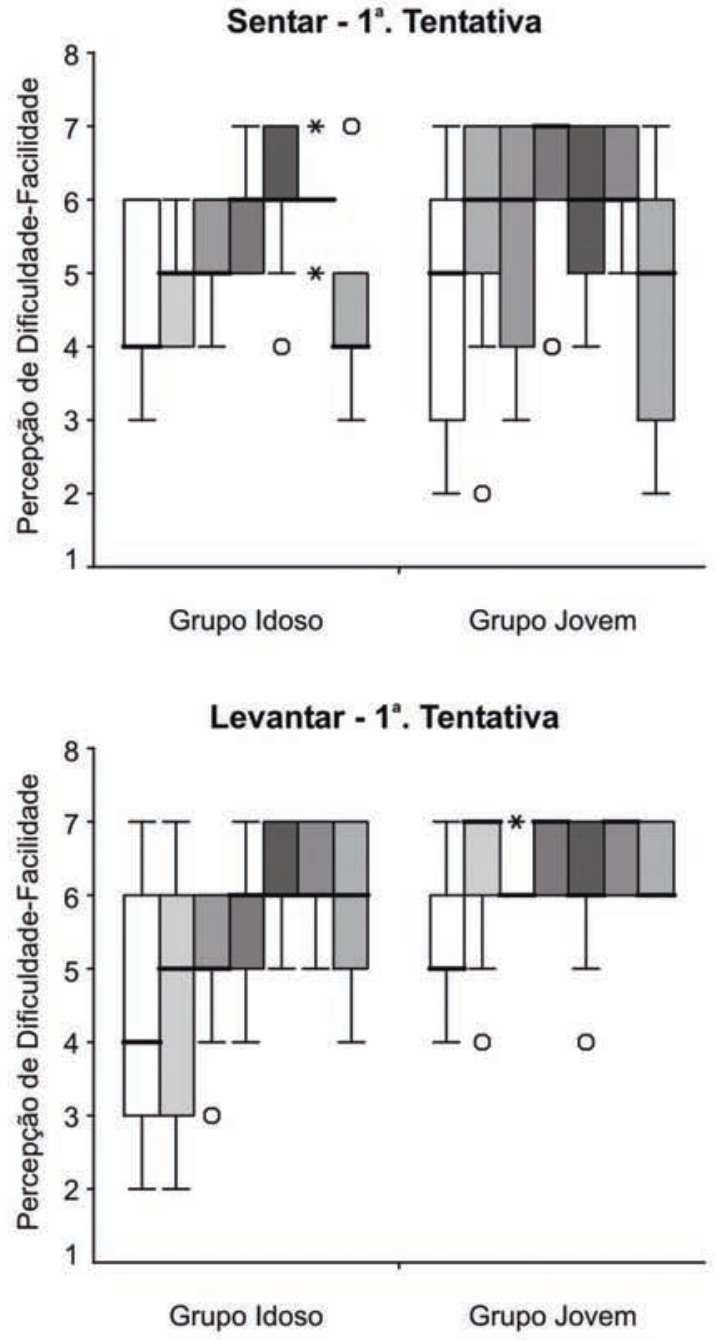

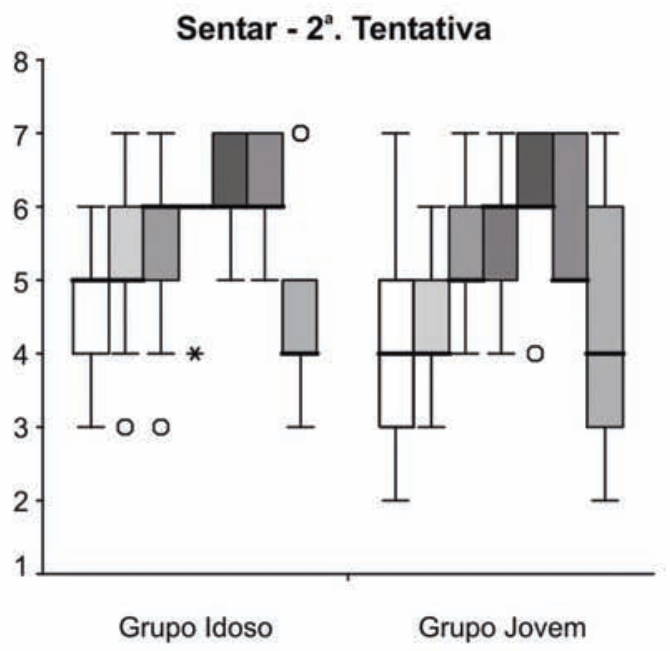

Levantar $-2^{\mathrm{a}}$. Tentativa

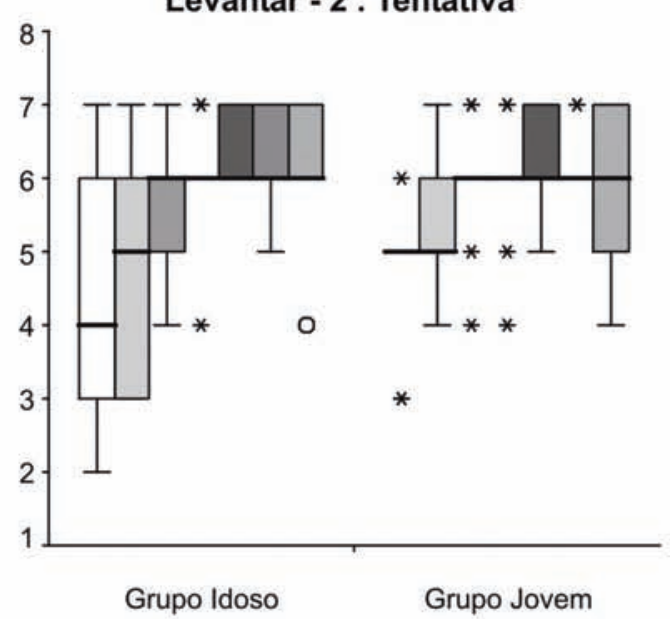

Grupo Idoso

Grupo Jovem

\section{$1 \square 2 \square 3$

Figura 4. Boxplots da percepção de dificuldade/facilidade das sete alturas analisadas. O símbolo o representa os casos com valores entre 1,5 e 3,0 vezes o comprimento do retângulo do boxplot a partir da margem inferior ou superior do retângulo. O símbolo * representa os casos com valores acima de 3,0 vezes o comprimento do retângulo do boxplot a partir da margem inferior ou superior do retângulo (GI: grupo idoso | GJ: grupo jovem).

aos indivíduos idosos $(M=0,69, S D=0,09)$. O coeficiente de determinação foi semelhante entre os dois grupos avaliados, jovens $(M=0,80, S D=0,17)$ e idosos $(M=0,77, S D=0,16)$.

\section{Discussão}

O presente estudo foi desenvolvido com o intuito de avaliar objetivamente os aspectos motores e perceptivos envolvidos na realização das tarefas de sentar e levantar de uma cadeira com diferentes alturas de assento e influências do processo de envelhecimento. Estudos anteriores focaram muita atenção nos componentes motores envolvidos, em especial no ato de levantar de uma cadeira, mas negligenciaram a relação da ação com a percepção oriunda da realização do movimento. Essa relação torna-se particularmente relevante no presente caso por envolver o processo de envelhecimento. Assim, a discussão que se segue abordará os aspectos motores e perceptivos e suas possíveis relações durante o processo de envelhecimento.

\section{Parâmetros motores das tarefas de sentar e levantar}

A análise da duração do movimento confirmou o que tem sido encontrado na literatura, ou seja, de modo geral indivíduos idosos sentam e levantam mais lentamente do que indivíduos jovens (Ikeda \& cols., 1991; Yoshida, Iwakura \& Inoue, 1983). Possivelmente essa maior lentidão seja resultado do próprio processo de envelhecimento que tem como consequências diminuição de força, equilíbrio, entre outras características (Spirduso, 1995). Por sua vez, o aumento da altura do assento gerou uma diminuição na duração do sentar e do levantar. Isso era esperado em função da diminuição da distância percorrida para sentar e levantar nas alturas mais altas do assento (Rodosky \& cols., 1989).

Os idosos, em geral, gastaram mais tempo na fase de abaixamento do sentar do que os jovens nas alturas baixas do assento, enquanto que nas alturas altas do assento o comportamento entre jovens e idosos foi similar. Esta diferença deve- 
se ao fato de que, durante o ato de sentar, os indivíduos são colocados diante de uma incerteza. Essa incerteza consiste no fato de sentar sobre um alvo (i.e., assento da cadeira) que não está disponível visualmente durante o deslocamento para baixo. O fato dos participantes olharem para frente durante o desempenho do movimento de sentar pode ter criado uma maior insegurança entre os idosos, que acabaram sentando mais lentamente para alcançar a meta da tarefa. Além disso, existe o efeito da ação da gravidade. Quando sentamos, a ação da gravidade nos empurra para baixo. Assim, indivíduos idosos podem estar sentando mais lentamente por conta da incerteza sobre a posição do assento e para não desabarem em cima da cadeira e correrem um risco maior de cair ou não amortecerem corretamente o gesto no momento do impacto com a superfície.

Durante a fase de estabilização do sentar, os idosos gastaram mais tempo nas alturas altas do assento em comparação aos adultos jovens, enquanto que a duração foi similar nas alturas baixas do assento. A fase de estabilização reflete prioritariamente ajustes finos na postura do corpo após o contato com o assento. $\mathrm{O}$ maior tempo gasto nas alturas mais altas do assento pode refletir uma menor estabilidade postural para manter a posição sentada nessas alturas, já que a área de contato do corpo com o assento diminui consideravelmente. Nas alturas mais baixas do assento, toda a nádega e parte das coxas dos participantes ficam em contato com o assento da cadeira. Nas alturas mais altas do assento, por sua vez, somente parte das nádegas fica em contato com o assento. Assim, após o contato com o assento, a fase de estabilização alonga-se para permitir o posicionamento adequado do tronco para garantir uma boa estabilidade corporal.

Os idosos demoram mais tempo para levantar durante a fase de geração do momento linear. Esse maior tempo para completar essa fase permite aumentar o impulso corporal, sem necessariamente aplicar mais força durante a execução do movimento. Existe uma relação biomecânica bem estabelecida entre impulso e momento. $\mathrm{O}$ aumento do impulso resultará em um aumento do momento linear. Por conta da ação da gravidade oposta ao movimento de levantar, é fundamental gerar um momento suficiente para sobrepor a força da gravidade e evitar retornar ao assento após iniciar o movimento de levantar. Em geral, o comportamento entre jovens e idosos é muito mais similar no levantar do que no sentar. No ato de levantar, a ação da gravidade é oposta, ou seja, o idoso tem que gerar uma força maior do que a gravidade para conseguir levantar e talvez por isso levante com uma duração mais próxima a do jovem. Se o idoso não conseguir gerar uma força suficiente para vencer a força da gravidade, será impossível levantar.

Um dado bastante significativo do presente estudo foi o aumento do número de unidades de movimento exibido pelos idosos na altura mais baixa do assento durante o movimento de sentar (Figura 3). Esse aumento reflete uma menor fluidez na realização do movimento, indicando ajustes constantes na trajetória dos segmentos e articulações analisados (Clearfield \& cols., 2006). Uma possível razão para o aumento desses ajustes na trajetória do movimento pode ser decorrente do efeito da força da gravidade que empurra o corpo para baixo. O sistema de controle motor deve garantir que o efeito da gravidade não faça com que a pessoa desabe sobre o assento da cadeira, pois isso poderia ter consequências graves para os idosos, em especial. O fluxo de informações sensoriais oriundas dos receptores envolvidos com a realização do movimento permite identificar se o movimento está sendo realizado como planejado ou não. No caso de desvios em relação ao planejamento inicial, ajustes na trajetória do movimento podem ser implementados para garantir a realização bem-sucedida do sentar. No presente caso, os ajustes constantes na trajetória do movimento são um indicativo de que os idosos realizam-no usando um modo de controle on-line. No controle on-line, a informação sensorial é usada para guiar a realização do movimento (Patla, 1997). Os adultos jovens, por outro lado, parecem depender menos do controle on-line para realizar o mesmo movimento, já que o realizam de forma mais suave e com melhor fluidez como apontado pela redução no número de unidades de movimento. Essa maior dependência do controle on-line sugere que os adultos idosos apresentam mais dificuldade para planejar corretamente a realização do movimento de sentar. Liu-Ambrose, Ahamed, Graf, Feldman e Robinovitch (2008) encontraram que idosos caidores ,com memória de trabalho piorada, superestimam seus limites de estabilidade, prejudicando o planejamento motor em tarefas da vida diária.

\section{Parâmetros perceptivos das tarefas de sentar e levantar}

Em geral, a análise dos resultados da escala de categorias não identificou diferenças entre os grupos avaliados sobre a percepção de dificuldade/facilidade para realizar os movimentos de sentar e levantar. Para o movimento de levantar foram observadas diferenças perceptivas entre os dois grupos somente para as alturas 2 e 3 do assento no primeiro bloco de tentativas, sendo que a tarefa de levantar foi julgada como sendo mais fácil pelos adultos jovens. A ausência de diferença entre jovens e idosos na maioria das alturas testadas e das tentativas realizadas contradiz os trabalhos de Munton e cols. (1981) e Wheeler e cols. (1985) que indicaram que os idosos relatam mais dificuldade para levantar quando comparados a adultos jovens. Essa inconsistência dos nossos dados com os dados de outros estudos pode ser resultado do fato de que os idosos participantes do presente estudo superestimaram suas capacidades de sentar e levantar do assento. Como apontado anteriormente, indivíduos idosos caidores superestimam seus limites de estabilidade quando solicitados a julgar a inclinação máxima do corpo à frente (Liu-Ambrose \& cols., 2008).

Os valores do coeficiente de determinação próximos de 1,0 indicam um bom relacionamento entre o estímulo físico e a resposta subjetiva. Dessa forma, o procedimento adotado no presente estudo para avaliar a percepção da posição articular foi satisfatório. Os indivíduos do GJ exibiram uma tendência de constância perceptiva, ou seja, a relação entre estímulo físico e resposta subjetiva mostrou ser linear. Os indivíduos idosos exibiram, por sua vez, uma tendência de subconstância perceptiva na tarefa de percepção da posição dos membros inferiores. Segundo DaSilva (1985), quando o expoente é menor do que 1,0, há uma aceleração negativa na função que relaciona o estímulo físico e a resposta estimada. O que ocorre é que os sujeitos têm uma tendência em julgar a proporção de 
aumento entre os estímulos com uma proporção menor ao longo do contínuo testado. Dessa forma, os jovens percebiam corretamente a magnitude dos incrementos na escala de estímulos, enquanto que os idosos, progressivamente com o aumento das alturas dos assentos, percebiam a articulação do joelho em amplitudes cada vez mais reduzidas, ou flexionadas, em relação à posição real.

Essa tendência à subconstância perceptiva por parte dos indivíduos idosos indica menor acurácia proprioceptiva. Essa perda proprioceptiva deve estar relacionada com o próprio processo de envelhecimento. Autores como Skinner e cols. (1984) e Kaplan e cols. (1985) têm apontado uma associação entre envelhecimento e declínio no sentido de posição articular. Possivelmente, durante o envelhecimento há uma perda da capacidade de obter informação proprioceptiva em função de déficits na estrutura do organismo. A perda funcional de receptores articulares, musculares e cutâneos pode estar provocando menor quantidade de informação disponível para o sujeito julgar sua posição articular.

\section{Relação entre percepção e ação nas tarefas de sentar e levantar}

No comportamento de sentar, e especialmente na altura mais baixa, houve similaridade dos julgamentos de dificuldade/facilidade entre jovens e idosos. Porém, a análise das unidades de movimento aponta o uso de mecanismos de controle diferentes para a altura baixa e para a altura alta do assento pelos indivíduos idosos. A alteração no mecanismo de controle não sensibilizou os idosos no sentido de interpretar essa tarefa (i.e., sentar na altura mais baixa) como sendo mais difícil. Considerando que o maior controle on-line do sentar na altura mais baixa é resultado do medo de cair sobre o assento, é estranho pensar que os idosos não perceberam que era mais difícil sentar nesta altura do que nas outras alturas do assento. $\mathrm{O}$ esperado seria o julgamento de dificuldade crescente com a diminuição da altura do assento para os indivíduos idosos.

Além disso, existe a questão da perda proprioceptiva. O déficit proprioceptivo pode justificar também os julgamentos discrepantes sobre dificuldade/facilidade dos idosos. Se há um empobrecimento na interpretação da posição dos segmentos corporais, consequentemente eles poderiam identificar uma altura muito baixa como não sendo tão baixa em comparação com outras.

\section{Considerações Finais}

Em síntese, podemos concluir que os indivíduos idosos exibiram mudanças com relação à estratégia de controle motor usada na realização do movimento de sentar em uma cadeira com alturas baixas de assento. Tais mudanças foram associadas com movimentos lentos e com um número elevado de unidades de movimento. Em termos perceptivos, os idosos apresentaram dificuldade para perceber seus segmentos no espaço durante a realização dos gestos de sentar e levantar. Apesar dessa diferença proprioceptiva, os indivíduos idosos julgaram o nível de dificuldade/facilidade de realização das tarefas de sentar e levantar da mesma forma que indivíduos jovens, sugerindo uma superestimação da sua capacidade de realizar a tarefa. Entretanto, a percepção de dificuldade/ facilidade da tarefa de sentar pelos idosos não concorda com o desempenho motor exibido pelos idosos na altura mais baixa do assento. Enquanto a modulação motora no sentar variou ao longo da diminuição de altura de assento, a percepção do grau de dificuldade na realização do gesto permaneceu relativamente inalterada.

\section{Referências}

Clearfield, M. W., Diedrich, F. J., Smith, L. B., \& Thelen, E. (2006). Young infants reach correctly in A-not-B tasks: On the development of stability and perseveration. Infant Behavior \& Development, 29, 435-444.

Coghlin, S. S., \& McFadyen, B. J. (1994). Transfer strategies used to rise from a chair in normal and low back pain subjects. Clinical Biomechanics, 9, 85-92.

DaSilva, J. A. (1985). Scales for perceived egocentric distance in a large open field: Comparison of three psychophysical methods. American Journal of Psychology, 98, 119-144.

DaSilva, J. A., \& Macedo, L. (1983). A função potência na percepção: significado e procedimentos de cálculo do expoente. Arquivos Brasileiros de Psicologia, 34, 27-45.

Gibson, J. J. (1979). The ecological approach to visual perception. Boston: Houghton Mifflin.

Hirschfeld, H., Thorsteinsdottir, M., \& Olsson, E. (1999). Coordinated ground forces exerted by buttocks and feet are adequately programmed for weight transfer during sit-to-stand. Journal of Neurophysiology, 82, 3021-3029.

Ikeda, E. R., Schenkman, M. L., Riley, P. O., \& Hodge, W. A. (1991). Influence of age on dynamics of rising from a chair. Physical Therapy, 71, 473-481.

Janssen, W. G., Bussmann, H. B., \& Stam, H. J. (2002). Determinants of the sit-to-stand movement: A review. Physical Therapy, 82, 866-879.

Kaplan, F. S., Nixon, J. E., Reitz, M., Rindfleish, L., \& Tucker, J. (1985). Age-related changes in proprioception and sensation of joint position. Acta Orthopaedica Scandinavica, 56, 72-74.

Kokmen, E., Bossemeyer, R. W. Jr., \& Williams, W. J. (1978). Quantitative evaluation of joint motion sensation in an aging population. Journal of Gerontology, 33, 62-67.

Liu-Ambrose, T., Ahamed, Y., Graf, P., Feldman, F., \& Robinovitch, S. N. (2008). Older fallers with poor working memory overestimate their postural limits. Archives of Physical Medicine and Rehabilitation, 89, 1335-1340.

Mark, L. S. (1987). Eyeheight-scaled information about affordances: A study of sitting and stair climbing. Journal of Experimental Psychology: Human Perception and Performance, 13, 361-370.

Mark, L. S., \& Vogele, D. (1987). A biodynamic basis for perceived categories of action: A study of sitting and stair climbing. Journal of Motor Behavior, 19, 367-384.

Moraes, R. (1999). Efeitos do envelhecimento nas habilidades de andar para frente, andar para trás, sentar e levantar. Dissertação de Mestrado, Universidade Estadual Paulista, Rio Claro. 
Mourey, F., Grishin, A., d’Athis, P., Pozzo, T., \& Stapley, P. (2000). Standing up from a chair as a dynamic equilibrium task: A comparison between young and elderly subjects. The Journals of Gerontology. Series A, Biological Sciences and Medical Sciences, 55, B425-B431.

Munton, J. S., Ellis, M. I., Chamberlain, M. A., \& Wright, V. (1981). An investigation into the problems of easy chairs used by the arthritic and the elderly. Rheumatology and Rehabilitation, 20, 164-173.

Munton, J. S., Ellis, M. I., \& Wright, V. (1984). Use of electromyography to study leg muscle activity in patients with arthritis and in normal subjects during rising from a chair. Annals of the Rheumatic Diseases, 43, 63-65.

Noble, B. J., \& Robertson, R. J. (1996). Perceived exertion. Champaign: Human Kinetics.

Nuzik, S., Lamb, R., VanSant, A., \& Hirt, S. (1986). Sit-tostand movement pattern: A kinematic study. Physical Therapy, 66, 1708-1713.

Pai, Y. C., \& Lee, W. A. (1994). Effect of a terminal constraint on control of balance during sit-to-stand. Journal of Motor Behavior, 26, 247-256.

Pai, Y. C., \& Rogers, M. W. (1990). Control of body mass transfer as a function of speed of ascent in sit-to-stand. Medicine and Science in Sports and Exercise, 22, 378-384.

Pai, Y. C., \& Rogers, M. W. (1991a). Segmental contributions to total body momentum in sit-to-stand. Medicine and Science in Sports and Exercise, 23, 225-230.

Pai, Y. C., \& Rogers, M. W. (1991b). Speed variation and resultant joint torques during sit-to-stand. Archives of Physical Medicine and Rehabilitation, 72, 881-885.

Patla, A. E. (1997). Understanding the roles of vision in the control of human locomotion. Gait \& Posture, 5, 54-69.

Patla, A. E., \& Shumway-Cook, A. (1999). Dimensions of mobility: Defining the complexity and difficulty associated with community mobility. Journal of Aging and Physical Activity, 7, 7-19.

Rodosky, M. W., Andriacchi, T. P., \& Andersson, G. B. (1989) The influence of chair height on lower limb mechanics during rising. Journal of Orthopaedic Research, 7, 266-271.
Schenkman, M., Berger, R. A., Riley, P. O., Mann, R. W., \& Hodge, W. A. (1990). Whole-body movements during rising to standing from sitting. Physical Therapy, 70, 638-648.

Schenkman, M., Hughes, M.A., Samsa, G., \& Studenski, S. (1996). The relative importance of strength and balance in chair rise by functionally impaired older individuals. Journal of the American Geriatrics Society, 44, 1441-1446.

Shumway-Cook, A., \& Woollacott, M. H. (2007). Motor control: Translating research into clinical practice. Philadelphia: Lippincott Williams \& Wilkins.

Skinner, H. B., Barrack, R. L., \& Cook, S. D. (1984). Agerelated decline in proprioception. Clinical Orthopaedics and Related Research, 184, 208-211.

Spirduso, W. W. (1995). Physical dimensions of aging. Champaign: Human Kinetics.

Stevens, S. S. (1975). Psychophysics: Introduction to its perceptual, neural and social prospects. New York: Wiley Interscience.

Tinetti, M. E., Speechley, M., \& Ginter, S. F. (1988). Risk factors for falls among elderly persons living in the community. The New England Journal of Medicine, 319, 1701-1707.

Wheeler, J., Woodward, C., Ucovich, R. L., Perry, J., \& Walker, J. M. (1985). Rising from a chair: Influence of age and chair design. Physical Therapy, 65, 22-26.

Winter, D. A. (2005). Biomechanics and motor control of human movement. New Jersey: Wiley.

Yoshida, K., Iwakura, H., \& Inoue, F. (1983). Motion analysis in the movements of standing up from and sitting down on a chair: A comparison of normal and hemiparetic subjects and the differences of sex and age among the normals. Scandinavian Journal of Rehabilitation Medicine, 15, 133-140.

Recebido em 03.09.2008

Primeira decisão editorial em 13.08.2009

Versão final em 24.08.2009

Aceito em 24.09.2009 